IPM/P-2002/014

\title{
Space-Time Symmetries, T-Duality and Gauge Theory
}

\author{
Davoud Kamani \\ Institute for Studies in Theoretical Physics and Mathematics (IPM) \\ P.O.Box: 19395-5531, Tehran, Iran \\ E-mail:kamani@theory.ipm.ac.ir
}

\begin{abstract}
In this paper we study $U(1)$ gauge transformations on the space-time coordinates and on the background fields $g_{\mu \nu}$ and $\phi$. For some special gauge functions, gauged coordinates and gauged $U(1)$ field are equivalent to the rotated coordinates and rotated gauge field. We find gauge transformations that are symmetries of the string action. Also we obtain general $T$-duality transformations for the background fields. For special background fields this duality is equivalent to a gauge transformation.
\end{abstract}

PACS: $11.25 .-\mathrm{w}$

Keywords: Gauge theory; String theory; T-duality. 


\section{Introduction}

As it has been mentioned by A.M. Polyakov, the relation between gauge fields and strings is very important [1]. In fact this relation is useful in solving the problem of gauge-stringspacetime correspondence. From the other side $T$-duality transformation is an exact symmetry of the closed string theory [2]. We shall concentrate on the gauge theory, string theory and $T$-duality. In the presence of a $U(1)$ gauge field, we study the gauge transformations of the spacetime coordinates, the metric $g_{\mu \nu}$ and the dilaton $\phi$. We compare these with some other transformations of the spacetime. In other words some of the transformations on the spacetime and gauge fields can be interpreted as gauge transformations. We find gauge transformations that are symmetries of the superstring action.

Previously we have studied a general $T$-duality [3]. By this, we find general $T$-duality transformations on the spacetime coordinates and on the NS $\otimes$ NS fields. For some special background fields this general $T$-duality is equivalent to a gauge transformation. In this case the above dual space is a gauged space. In this gauged space we find the effective field strength that can be seen by a closed string.

In the second section, we shall study gauge transformations of the spacetime and their effects on the string action. In the third section, using the above general $T$-duality, we concentrate on the gauge-string relations.

\section{Gauge transformations of the spacetime}

A $U(1)$ gauge field $A_{\mu}$ with the constant field strength $F_{\mu \nu}$ can be written as

$$
A_{\mu}=-\frac{1}{2} F_{\mu \nu} X^{\nu}
$$

Let $\operatorname{det} F_{\mu \nu} \neq 0$, therefore

$$
X^{\mu}=-2 G^{\mu \nu} A_{\nu}
$$

where $G=F^{-1}$. According to this equation we can perform gauge transformation on the spacetime coordinates. Under the gauge transformation

$$
A_{\mu} \rightarrow \tilde{A}_{\mu}=A_{\mu}+\partial_{\mu} \Lambda,
$$

the coordinate $X^{\mu}$ has the following transformation

$$
X^{\mu} \rightarrow \tilde{X}^{\mu}=X^{\mu}-2 G^{\mu \nu} \partial_{\nu} \Lambda .
$$


Therefore the gauge field $\tilde{A}_{\mu}$ is

$$
\tilde{A}_{\mu}=-\frac{1}{2} F_{\mu \nu} \tilde{X}^{\nu}
$$

The equation (4) implies a transformation $X^{\mu} \rightarrow \tilde{X}^{\mu}=M^{\mu}{ }_{\nu} X^{\nu}+a^{\mu}$ with the condition $F M+M^{T} F=2 F$, can be interpreted as a gauge transformation in the spacetime with the gauge function

$$
\Lambda=\frac{1}{4} \sigma_{\mu \nu} X^{\mu} X^{\nu}-\frac{1}{2} F_{\mu \nu} X^{\mu} a^{\nu}+\Lambda_{0}
$$

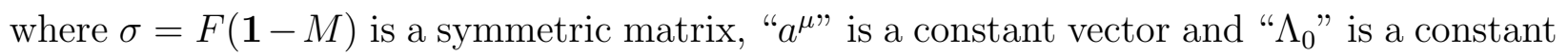
number. Any translation $\delta X^{\mu}=a^{\mu}$ (i.e. $M=\mathbf{1}$ ) corresponds to a gauge transformation in the spacetime. If the matrix $M$ is antisymmetric, the gauge function (6) describes Lorentz transformations.

If the gauge function $\Lambda$ satisfies the equation

$$
2\left(G^{2}\right)^{\mu \nu} \partial_{\mu} \Lambda \partial_{\nu} \Lambda+G^{\mu \nu} J_{\mu \nu} \Lambda=0
$$

where $J_{\mu \nu}$ is the operator $J_{\mu \nu}=X_{\mu} \partial_{\nu}-X_{\nu} \partial_{\mu}$, the square length $X^{\mu} X_{\mu}$ remains invariant. If $a^{\mu}=0$ and the matrix $M$ is orthogonal, as expected the gauge function (6) satisfies this equation.

For a special gauge function $\Lambda$, it is possible to write $\tilde{X}^{\mu}$ as a linear combination of $\left\{X^{\mu}\right\}$. Let the matrix $S_{\mu \nu}$ be symmetric and constant. For the gauge function

$$
\Lambda=\frac{1}{4} S_{\mu \nu} X^{\mu} X^{\nu}+\Lambda_{0}
$$

the gauge transformed coordinates are

$$
\tilde{X}^{\mu}=(\mathbf{1}-G S)^{\mu}{ }_{\nu} X^{\nu}
$$

Now consider a rotation in the spacetime

$$
X_{(r)}^{\mu}=\mathcal{A}_{\nu}^{\mu} X^{\nu}
$$

We do not restrict the matrix $\mathcal{A}$ to be orthogonal. The gauge field $A_{\mu}$ transforms as

$$
A_{(r)}^{\mu}=-\frac{1}{2}\left[\left(\mathcal{A}^{-1}\right)^{T} F\right]_{\mu \nu} X^{\nu}
$$

Equality of the rotated and gauge transformed versions of the field $A_{\mu}$ leads to the equation

$$
\mathcal{A}^{-1}=\mathbf{1}+G S
$$


In addition if we impose the equality of the gauged space and the rotated one, we obtain

$$
\mathcal{A}=\mathbf{1}-G S .
$$

Therefore the matrices $S$ and $G$ should satisfy the condition

$$
S G S=0 .
$$

Thus we choose those gauge transformations with $\operatorname{det} S=0$, i.e. the matrix $S$ is not invertible. For example for two dimensional spacetime the matrix $S$ has the form

$$
S=\left(\begin{array}{cc}
a & \pm \sqrt{a b} \\
\pm \sqrt{a b} & b
\end{array}\right) .
$$

For arbitrary $a$ and $b$ with $a b \geq 0$ this matrix satisfies the condition (14).

Superstring in the gauged spacetime

Now let the set $\left\{X^{\mu}\right\}$ denote the string coordinates. The action of string that propagates in the background fields $g_{\mu \nu}, A_{\mu}$ and the dilaton $\phi$, is [5]

$$
\begin{aligned}
S= & -\frac{1}{4 \pi \alpha^{\prime}} \int d^{2} \sigma \sqrt{-h}\left(h^{a b} g_{\mu \nu} \partial_{a} X^{\mu} \partial_{b} X^{\nu}-\frac{\epsilon^{a b}}{\sqrt{-h}} F_{\mu \nu} \partial_{a} X^{\mu} \partial_{b} X^{\nu}\right. \\
& \left.+\alpha^{\prime} R \phi(X)\right) .
\end{aligned}
$$

Consider the gauge transformations of $g_{\mu \nu}$ and $\phi$ as

$$
\begin{aligned}
& g_{\mu \nu} \rightarrow \tilde{g}_{\mu \nu}, \\
& \phi \rightarrow \tilde{\phi} .
\end{aligned}
$$

Invariance of the action (16) under the gauge transformations (9) and (17), leads to the equations

$$
\begin{aligned}
& \mathcal{A}^{T} \tilde{g} \mathcal{A}=g, \\
& \mathcal{A}^{T} F \mathcal{A}=F, \\
& \tilde{\phi}=\phi .
\end{aligned}
$$

The set of matrices $\{\mathcal{A}\}$ that satisfy the equation $\mathcal{A}^{T} F \mathcal{A}=F$, form a group. According to the equation (13), the second equation also gives the condition (14).

The metric of the gauged space is

$$
\tilde{g}_{\mu \nu}=g_{\mu \nu}+\left(G S-S G-S G^{2} S\right)_{\mu \nu} .
$$


The second part purely is the effect of the chosen gauge.

Worldsheet supersymmetry gives the gauged fermions as

$$
\tilde{\psi}_{ \pm}^{\mu}(\tau \pm \sigma)=(\mathbf{1}-G S)^{\mu}{ }_{\nu} \psi_{ \pm}^{\nu}(\tau \pm \sigma) .
$$

These are components of the fermions on the superstring worldsheet, that lives in the gauged spacetime. The first equation of (18) implies the fermionic term of the superstring action, i.e. $g_{\mu \nu} \bar{\psi}^{\mu} \rho^{a} \partial_{a} \psi^{\nu}$ is gauge invariant. Therefore the superstring action is symmetric under the gauge transformations (9) and (18).

If some of the spacetime coordinates $\left\{X^{\bar{\mu}}\right\}$ are compact on a tori with the radii $\left\{R_{\bar{\mu}}\right\}$, we have

$$
\begin{aligned}
& X^{\bar{\mu}}(\sigma+\pi, \tau)-X^{\bar{\mu}}(\sigma, \tau)=2 \pi L^{\bar{\mu}}=2 \pi n^{\bar{\mu}} R_{\bar{\mu}}, \\
& X^{\prime \bar{\mu}}(\sigma+\pi, \tau)-X^{\prime \bar{\mu}}(\sigma, \tau)=2 \pi \alpha^{\prime} p^{\bar{\mu}}=2 \pi m^{\bar{\mu}} \frac{\alpha^{\prime}}{R_{\bar{\mu}}},
\end{aligned}
$$

where $X^{\prime \bar{\mu}}=X_{L}^{\bar{\mu}}-X_{R}^{\bar{\mu}}$ is $T$-dual coordinate of $X^{\bar{\mu}}$. The sets $\left\{n^{\bar{\mu}}\right\}$ and $\left\{m^{\bar{\mu}}\right\}$ are winding and momentum numbers of the closed string around the compact directions $\left\{X^{\bar{\mu}}\right.$. For the gauged coordinates we obtain

$$
\begin{aligned}
& \tilde{X}^{\bar{\mu}}(\sigma+\pi, \tau)-\tilde{X}^{\bar{\mu}}(\sigma, \tau)=2 \pi \tilde{L}^{\bar{\mu}}, \\
& \tilde{X}^{\prime \bar{\mu}}(\sigma+\pi, \tau)-\tilde{X}^{\prime \bar{\mu}}(\sigma, \tau)=2 \pi \alpha^{\prime} \tilde{p}^{\bar{\mu}},
\end{aligned}
$$

where

$$
\begin{aligned}
& \tilde{L}^{\bar{\mu}}=(\mathbf{1}-G S)^{\bar{\mu}}{ }_{\bar{\nu}} L^{\bar{\nu}}, \\
& \tilde{p}^{\bar{\mu}}=(\mathbf{1}-G S)^{\bar{\mu}}{ }_{\bar{\nu}} p^{\bar{\nu}} .
\end{aligned}
$$

If $\tilde{L}^{\bar{\mu}}$ is not zero, the coordinate $\tilde{X}^{\bar{\mu}}$ of the gauged space also is compact. Consider the case that the gauged space is non-compact, i.e. $\tilde{L}^{\bar{\mu}}=0$ for any $\bar{\mu}$. This leads to the condition $\operatorname{det}(\mathbf{1}-G S)=0$, which does not hold. Therefore if some directions of the spacetime are compact, always some coordinates of the gauged space also are compact.

\section{General $T$-duality and gauge theory}

Consider the following transformations on the left and right moving parts of the compact coordinates $\left\{X^{\bar{\mu}}\right\}$

$$
\begin{aligned}
& X_{L}^{\bar{\mu}}(\tau+\sigma) \rightarrow Y_{L}^{\bar{\mu}}(\tau+\sigma)=\mathcal{B}^{\bar{\mu}}{ }_{\nu} X_{L}^{\bar{\nu}}(\tau+\sigma), \\
& X_{R}^{\bar{\mu}}(\tau-\sigma) \rightarrow Y_{R}^{\bar{\mu}}(\tau-\sigma)=-\mathcal{B}^{\bar{\mu}}{ }_{\bar{\nu}} X_{R}^{\bar{\nu}}(\tau-\sigma) .
\end{aligned}
$$


From these transformations we obtain

$$
\begin{aligned}
& X^{\bar{\mu}} \rightarrow Y^{\bar{\mu}}=\mathcal{B}^{\bar{\mu}}{ }_{\bar{\nu}} X^{\prime \bar{\nu}}, \\
& X^{\prime \bar{\mu}} \rightarrow Y^{\prime \bar{\mu}}=\mathcal{B}^{\bar{\mu}}{ }_{\bar{\nu}} X^{\bar{\nu}} .
\end{aligned}
$$

Therefore the compact part of the spacetime and its $T$-dual, transform to each other. For $\mathcal{B}=\mathbf{1}$ these are usual $T$-duality transformations.

Worldsheet supersymmetry enables us to obtain transformations of the worldsheet fermions,

$$
\begin{aligned}
& \psi_{+}^{\bar{\mu}} \rightarrow \chi_{+}^{\bar{\mu}}=\mathcal{B}^{\bar{\mu}}{ }_{\bar{\nu}} \psi_{+}^{\bar{\nu}}, \\
& \psi_{-}^{\bar{\mu}} \rightarrow \chi_{-}^{\bar{\mu}}=-\mathcal{B}^{\bar{\mu}}{ }_{\nu} \psi_{-}^{\bar{\nu}} .
\end{aligned}
$$

In other words we have the exchange transformations

$$
\begin{aligned}
& \psi^{\bar{\mu}} \rightarrow \chi^{\bar{\mu}}=\mathcal{B}^{\bar{\mu}}{ }_{\bar{\nu}} \psi^{\prime \bar{\nu}}, \\
& \psi^{\prime \bar{\mu}} \rightarrow \chi^{\prime \bar{\mu}}=\mathcal{B}^{\bar{\mu}}{ }_{\bar{\nu}} \psi^{\bar{\nu}} .
\end{aligned}
$$

That is, the worldsheet fermions $\left\{\psi^{\bar{\mu}}\right\}$ and their $T$-dual fermions $\left\{\psi^{\prime \bar{\mu}}\right\}$ are exchanged.

The invariance of the mass spectrum of the closed superstring leads to the fact that the matrix $\mathcal{B}$ is orthogonal

$$
\mathcal{B}^{T} \mathcal{B}=\mathcal{B B}^{T}=1
$$

Compactification of the $\left\{X^{\bar{\mu}}\right\}$ directions leads to the compactification of the coordinates $\left\{Y^{\bar{\mu}}\right\}$. Using the equations (21), gives

$$
\begin{aligned}
& Y^{\bar{\mu}}(\sigma+\pi, \tau)-Y^{\bar{\mu}}(\sigma, \tau)=2 \pi \Lambda^{\bar{\mu}}, \\
& Y^{\prime \bar{\mu}}(\sigma+\pi, \tau)-Y^{\prime \bar{\mu}}(\sigma, \tau)=2 \pi \alpha^{\prime} \Pi^{\bar{\mu}},
\end{aligned}
$$

where $\Pi^{\bar{\mu}}$ and $\Lambda^{\bar{\mu}}$ are

$$
\begin{gathered}
\Pi^{\bar{\mu}}=\frac{1}{\alpha^{\prime}} \mathcal{B}^{\bar{\mu}} L_{\bar{\nu}}^{\bar{\nu}} \\
\frac{1}{\alpha^{\prime}} \Lambda^{\bar{\mu}}=\mathcal{B}^{\bar{\mu}}{ }_{\bar{\nu}} p^{\bar{\nu}}
\end{gathered}
$$

These equations imply the momentum and the winding numbers of closed string in the Y-space depend on the winding and momentum numbers of it in the compact part of the spacetime respectively. 
Previously we have observed that for an emitted closed string from a $\mathrm{D}_{p}$-brane with the background fields $A_{\mu}$ and $B_{\mu \nu}$ (the $\mathrm{NS} \otimes \mathrm{NS}$ field), there is the following relation between its momentum and winding numbers [4]

$$
p^{\alpha}=-\frac{1}{\alpha^{\prime}} \mathcal{F}_{\beta}^{\alpha} L^{\beta},
$$

where $\mathcal{F}_{\mu \nu}=F_{\mu \nu}-B_{\mu \nu}$ is total field strength and the indices $\alpha$ and $\beta$ show the brane directions. According to this relation we have

$$
\frac{1}{\alpha^{\prime}} \Lambda^{\bar{\alpha}}=-\left(\mathcal{B F F}^{T}\right)^{\bar{\alpha}}{ }_{\bar{\beta}} \Pi^{\bar{\beta}}
$$

Comparing this equation with the relation (31) implies, in the Y-space closed string feels the total field strength $\mathcal{B F}^{-1} \mathcal{B}^{T}$.

Transformation of the string action

The action of a string that propagates in the background fields $g_{\mu \nu}, \mathcal{F}_{\mu \nu}$ and $\phi$, is [5]

$$
\begin{aligned}
S= & -\frac{1}{4 \pi \alpha^{\prime}} \int d^{2} \sigma \sqrt{-h}\left(h^{a b} g_{\mu \nu} \partial_{a} X^{\mu} \partial_{b} X^{\nu}-\frac{\epsilon^{a b}}{\sqrt{-h}} \mathcal{F}_{\mu \nu} \partial_{a} X^{\mu} \partial_{b} X^{\nu}\right. \\
& \left.+\alpha^{\prime} R \phi(X)\right) .
\end{aligned}
$$

In addition to the general $T$-duality transformations (25), consider the following transformations for the background fields

$$
\begin{aligned}
& g_{\mu \nu} \rightarrow \bar{g}_{\mu \nu}, \\
& \mathcal{F}_{\mu \nu} \rightarrow \overline{\mathcal{F}}_{\mu \nu}, \\
& \phi \rightarrow \bar{\phi} .
\end{aligned}
$$

Assume that all directions of the spacetime are compact on tori. Therefore the action (33) transforms as

$$
\begin{aligned}
\bar{S}= & -\frac{1}{4 \pi \alpha^{\prime}} \int d^{2} \sigma \sqrt{-h}\left(h^{a b}\left(\mathcal{B}^{T} \bar{g} \mathcal{B}\right)_{\mu \nu} \partial_{a} X^{\prime \mu} \partial_{b} X^{\prime \nu}-\frac{\epsilon^{a b}}{\sqrt{-h}}\left(\mathcal{B}^{T} \overline{\mathcal{F}} \mathcal{B}\right)_{\mu \nu} \partial_{a} X^{\prime \mu} \partial_{b} X^{\prime \nu}\right. \\
& \left.+\alpha^{\prime} R \bar{\phi}\left(X^{\prime}\right)\right) .
\end{aligned}
$$

Invariance of the action under the transformations (25) and (34) leads to the relations

$$
\begin{aligned}
& \mathcal{B}^{T} \bar{g} \mathcal{B}=g^{\prime}, \\
& \mathcal{B}^{T} \overline{\mathcal{F}} \mathcal{B}=\mathcal{F}^{\prime}, \\
& \bar{\phi}=\phi^{\prime},
\end{aligned}
$$


where $g_{\mu \nu}^{\prime}, \mathcal{F}_{\mu \nu}^{\prime}$ and $\phi^{\prime}$ denote the usual $T$-duality of these background fields. These equations give $\bar{g}=\mathcal{B} g^{\prime} \mathcal{B}^{T}$ and $\overline{\mathcal{F}}=\mathcal{B F}^{\prime} \mathcal{B}^{T}$. Therefore $\bar{g}_{\mu \nu}, \overline{\mathcal{F}}_{\mu \nu}$ and $\bar{\phi}$ are general $T$-duality transformations of the background fields, that are consistent with the equations (25). In fact the equations (36) change the action (35) to the $T$-dual form of the action (33).

We know that under the gauge transformations the total field strength $\mathcal{F}$ is invariant. We are interested in the case that above general $T$-duality to be a gauge transformation. In this case $\overline{\mathcal{F}}$ is proportional to $\mathcal{F}$, i.e.

$$
\overline{\mathcal{F}}_{\mu \nu}=-\frac{1}{\lambda} \mathcal{F}_{\mu \nu}
$$

where $\lambda$ is a constant number. The $T$-duality of the total field strength $\mathcal{F}$ is [6]

$$
\mathcal{F}^{\prime}=-(g+k \mathcal{F})^{-1} \mathcal{F}(g-k \mathcal{F})^{-1}
$$

where $k= \pm 1$. According to the equations (36)-(38) we can obtain the matrix $\mathcal{B}$

$$
\mathcal{B}=\ell \sqrt{\lambda} \Gamma(g-k \mathcal{F})^{-1}
$$

where $\ell= \pm 1$. The matrix $\Gamma$ is limited as the following

$$
\Gamma^{T} \mathcal{F} \Gamma=\mathcal{F}
$$

The orthogonality of the matrix $\mathcal{B}$ leads to the orthogonality of the matrix $\Gamma$

$$
\Gamma^{T} \Gamma=\Gamma \Gamma^{T}=\mathbf{1}
$$

and an additional condition on the background fields

$$
g^{2}-\mathcal{F}^{2}=\lambda \mathbf{1}
$$

This condition means that only some special background fields admit the equation (37) to hold. The set of orthogonal $\Gamma$ matrices that satisfy the equation $(40)$ form group $O(d)$, where " $d$ " is the spacetime dimension.

Since $T$-duality of the metric $g_{\mu \nu}$ is $g^{\prime}=(g+k \mathcal{F})^{-1} g(g-k \mathcal{F})^{-1}$, the equations (39) and (42) give the metric $\bar{g}$ as

$$
\bar{g}=\frac{1}{\lambda} \Gamma g \Gamma^{T}
$$

This is gauge transformed version of the spacetime metric. For $\Gamma=\mathbf{1}$ and $\lambda=-1$ the theory is gauge invariant and $\bar{g}=-g$. That is the signature of the metric is changed.

According to the equation (39), the relation (32) can be written as

$$
\frac{1}{\alpha^{\prime}} \Lambda^{\alpha}=\lambda\left(\Gamma \mathcal{F}^{\prime} \Gamma^{T}\right)_{\beta}^{\alpha} \Pi^{\beta}
$$

That is, in the gauged space closed string feels the effective field strength $-\frac{1}{\lambda} \Gamma \mathcal{F}^{\prime-1} \Gamma^{T}$. 


\section{Conclusion}

We observed that in the presence of a $U(1)$ gauge field with constant field strength, it is possible to perform gauge transformations on the spacetime coordinates, the metric $g_{\mu \nu}$ and the dilaton $\phi$. There are some gauge transformations that preserve the spacetime distances. Some rotations and translations in the spacetime are equivalent to some gauge transformations. For example Lorentz transformation is one of them. We showed that the string action

under a special gauge transformation is invariant. In addition we saw compactification of the spacetime is induced to the gauged space.

We showed that there is a generalization of the $T$-duality that preserves the compactification of the spacetime. In this duality the winding and momentum numbers of closed string get exchange. Invariance of the string action with the $\mathrm{NS} \otimes \mathrm{NS}$ background fields under the general $T$-duality gives general $T$-duality transformations of these fields. For special background fields the above general $T$-duality transformations are equivalent to gauge transformations. In this gauged space closed string probes a modified field strength.

\section{References}

[1] A.M. Polyakov, "Gauge Fields and Space-Time", hep-th/0110196.

[2] M. Dine, P. Huet and N. Seiberg, Nucl. Phys.B322(1989)301.

[3] D. Kamani, Nucl. Phys. B601(2001)149-168, hep-th/0104089.

[4] H. Arfaei and D. Kamani, Phys. Lett.B452(1999)54, hep-th/9909167.

[5] R.G. Leigh, Mod. Phys. Lett.A4 28(1989)2767.

[6] A. Giveon, M. Porrati, E. Rabinovici, Phys. Rep. 244(1994)77-202. 